\title{
Engaging the Periphery for Visual Communication on Mobile Phones
}

\author{
Lisa Cowan, William G. Griswold, Louise Barkhuus, James D. Hollan \\ University of California, San Diego \\ lgcowan@cs.ucsd.edu,.wgg@cs.ucsd.edu,barkhuus@cs.ucsd.edu, hollan@cogsci.ucsd.edu
}

\begin{abstract}
While mobile phones have become ubiquitous instruments of communication and social interaction, they still require explicit interaction, placing high demands on attention. Engaging the periphery of users' attention offers opportunities for awareness and interaction while reducing demands on attention and risks of disruption. We explore the mobile peripheral design space with Emotipix, an application for camera phones that turns the background of the phone's display into a place for visual conversations. We conducted an exploratory 2-week user study with 6 pairs and one 4person group, and found that Emotipix facilitated ongoing social practices. Our study shows that there is an unexploited opportunity to use mobile phones for peripheral awareness. We provide recommendations for managing users' expectations, desires for control, and privacy in mobile peripheral display design.
\end{abstract}

\section{Introduction}

Mobile phones have become ubiquitous; sophisticated camera phones that support taking and sharing photos are common in most of the developed world. The communication mechanisms (e.g., voice, SMS, MMS) now typical on these phones require explicit interaction, yet mobile phones also present opportunities for implicit interaction. People carry their phones with them nearly everywhere and interact with them regularly, and these devices could subtly provide additional information in the periphery during these interactions, without causing undue distraction.

In this paper, we explore how to engage the periphery of users' attention for visual communication on mobile phones. Although peripheral displays have been studied extensively $[10,15,26]$, few studies have looked closely at mobile peripheral displays $[4,11]$ and social practices with mobile peripheral displays have yet to be investigated.

We investigate these practices with Emotipix, an application that turns the background (i.e., wallpaper) of the phone's home screen into a place for conversations. Most phones support a wallpaper image, which users can explicitly set, and which typically serves a decorative purpose. Here, we explore appropriating this underutilized 'screen real estate' to facilitate social awareness and visual communication in the periphery.

Engaging both the periphery and the center of attention is an essential component of Weiser's seminal vision of ubiquitous computing. He defines the periphery as what we are attuned to without explicitly attending to and uses it to motivate 'calm technology', systems that engage both the center and the periphery of one's attention and allow ready movement between them [37]. Such systems allow flexibly attending to information without becoming overloaded. Further, Weiser argues that calm technology can create a sense of connectedness with the world and provide new opportunities for social interaction. Although it has been argued that calm computing should not be pursued to the exclusion of more engaging experiences [30], we contend that mobile peripheral displays have the potential to provide users with useful non-intrusive awareness as a natural component of phone use.

Emotipix aims to engage the periphery to support social awareness and conversation on mobile phones without causing information overload or disruption. It is designed to achieve these goals by placing the display in the background of the workflow, providing options for interaction without demanding their use, and requiring minimal explicit actions from the user. Emotipix displays an automated slide show of shared photos in the background wallpaper of the phone's home screen. When users take pictures with their camera phones they are automatically shared with their Emotipix buddies, small groups of friends or family. The photos then appear as wallpaper on their phones. A user can tap an icon on their phone's home screen to send karma, positive feedback about a photo. Emotipix simplifies photo viewing to glancing at your phone, photo sharing to simply taking a photo, and feedback to a single tap gesture.

In the remainder of this paper, we document how users interact with and through Emotipix. We examined the use of Emotipix for two weeks and present the results of this in-situ user study. We discuss how participants incorporated this new communication mechanism into their social practices and summarize design recommendations for effectively engaging the periphery for social awareness, connectedness, and communication on mobile phones. 


\section{Background and related work}

\subsection{Peripheral displays}

Computer-based peripheral displays have been investigated for their ability to convey numerous types of information [3, 23, 25]. Many of these systems present social information on large public displays and desktop displays $[10,15]$. Other systems have used home displays to enable awareness of family members. The Whereabouts Clock displays family members' locations [1], and Astra displays photos, text, and drawings sent from mobile devices [24]. In the Digital Family Portrait [26], awareness of family members' activities provides a sense of well-being.

Emotipix is particularly inspired by the KAN-G framework, which explores the notion of affective awareness, defined as a sense of being in touch with friends or family [21]. KAN-G provides channel-based publish/subscribe photo sharing with networked digital cameras. Displays are placed in the kitchen and other dwelling spots, and buttons on the display (e.g., “Applause!") enable spontaneous feedback. This system highlights the importance of peripheral awareness and feedback in social interaction.

Dey and Guzman's presence displays and the work by Kuwabara et al. on connectedness-oriented communication have shown that peripheral displays can foster social awareness and connectedness [9, 20]. Kaye's work on low-bandwidth communication has found that even a single click is meaningful when interpreted in the context of an intimate relationship [17]. Emotipix similarly aims to foster social awareness and connectedness through visual, photobased communication and 1-click feedback.

Some recent research has explored using mobile peripheral displays. Ubifit Garden employs a glanceable display in the background wallpaper of a mobile phone to encourage physical activity [4], and Ubigreen similarly provides a glanceable mobile display to support green transportation habits [11]. Emotipix explores using mobile peripheral displays for social awareness and communication, rather than personal information awareness. Since mobile phones already play a significant role in our social and communicative practices they provide an ideal platform for studying visual communication in the periphery.

\subsection{Photo sharing}

Much research has focused on digital photo sharing. Frohlich et al.'s study of photo practices has revealed a need for technological support for social uses of digital photos and for easier remote sharing and feedback [12]. Kindberg et al.'s study of camera phone use has also confirmed the need for easier sharing in the moment [18]. Okabe's study of camera phone usage in Japan notes that people share many personal and mundane photos, and that camera phones have changed what people consider photo-worthy [28, 29]. Voida has explored the ways that people appropriate photos for communication in an online chat system [36] and Sit et al. have explored photos as conversational anchors [31].

Picture messaging (MMS) provides a channel for mobile visual communication, yet the content must be consciously formed and addressed to specific recipients, and the required effort may deter use [18]. Recipients likewise must explicitly open the messages one-by-one and consciously form replies when desired. Radar, SLAM, and Flickr (www.flickr.com) employ a group-based publish/subscribe model for photo messaging, thus removing the overhead of addressing specific recipients [8, 13]. Flipper and Zurfer reduce barriers to mobile photo sharing by automating photo uploads and/or downloads, as in Emotipix [7, 27, 34]. These tools, as regular phone applications, still require explicit, conscious interaction to use, e.g., one must open the application window or select pictures to view. Emotipix in contrast, employs peripheral displays of shared photos and a lightweight feedback mechanism.

\subsection{Mobile social awareness}

Many researchers are exploring mobile social interaction. Taylor's study of SMS gift-giving practices among teenagers illustrates how social practices and new technologies evolve [33]. The study of Houston has gathered requirements for designing systems that provide awareness and social support to motivate physical activity [6]. Also, commercial mobile applications such as Facebook (www.facebook.com) and Twitter (www.twitter.com) now play major roles in providing ongoing social awareness.

Other work has explored how location-based technologies can enable new communication mechanisms and greater social awareness. For example, the study of Reno has examined social practices of location disclosure [32], and the study of Connecto has investigated how location and status sharing can help sustain group bonds [1].

\section{Emotipix}

\subsection{Engaging the periphery on mobile phones}

Mobile phones present opportunities for engaging the periphery for visual communication. People take their phones with them everywhere and look at them regularly. These ubiquitous, personal devices have the 


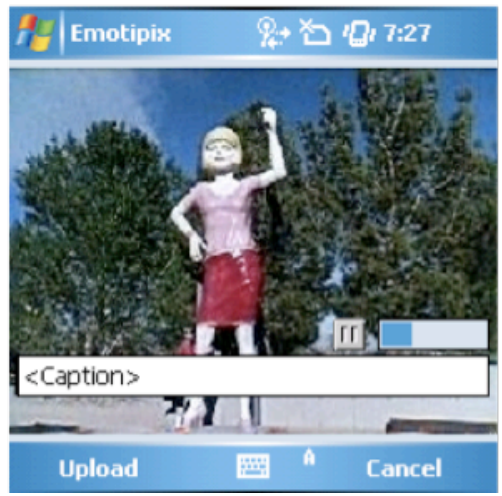

(a)

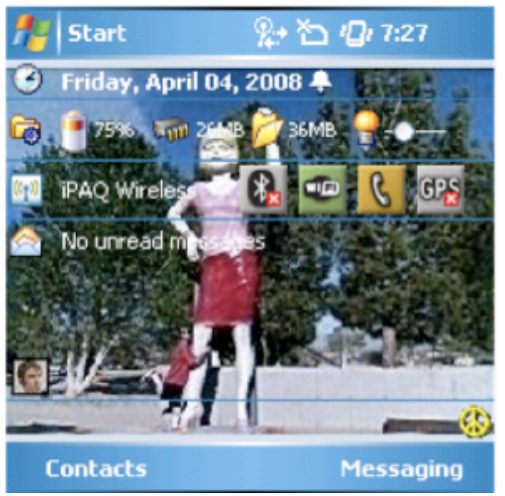

(b)

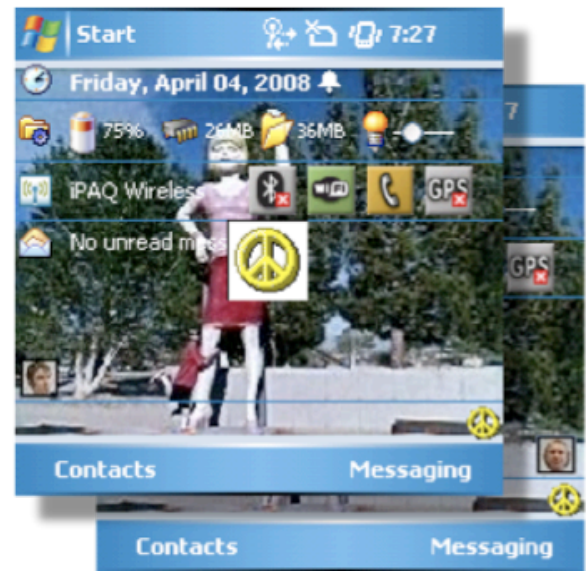

(c)

Figure 1. (a) Photos are uploaded automatically, unless cancelled. (b) Photos appear in the home screen's background. The publisher's sticker is at lower left; the Emotipix peace sign icon is at lower right. (c) Top: Tapping the Emotipix icon sends karma to a photo. A peace sign appears briefly to confirm. Bottom: After, the sender's sticker is at lower right.

capability to capture and display images and to exchange them over communication networks.

There are, however, a number of peripheral engagement challenges specific to mobile phones. First, the phone's display may be out of sight or blanked to save power. To make information available in the periphery despite the display's intermittent visibility, this information should be visible when the user turns on the display. Second, the phone's display is small, which demands careful use of the limited peripheral display space. Third, the context in which people use their phones is dynamic; people may be engaged in activities that place variable demands on their time and attention. To accommodate this variability, the interface should make information and interaction opportunities available without requiring that users attend to them. Also, the interface should facilitate anytime / anywhere access, despite lapses in network connectivity.

\subsection{The Emotipix approach}

Emotipix offers users options for passive, lightweight, or extended interaction:

Emotipix's peripheral display is placed in the background of the workflow, providing users with the option for passive interaction. People carry mobile phones around and use them frequently throughout the day. The wallpaper image is visible on the phone's home screen at the initiation of use and again when the user ends an activity on the phone. Because the wallpaper is visible in the display's background as users initiate and transition between tasks, it is an opportune place to present peripheral information.
Emotipix users have the option for lightweight interaction. Of course, a peripheral display must function well when used passively, yet it is important to provide a channel for lightweight feedback within the system. User feedback can complete the communication loop between photographers and viewers and can also be used to train the system to optimize content delivery to users' preferences [12]. To encourage feedback, yet preserve the user's ability to quickly shift attention between the peripheral display and the primary activity, we advocate a single-gesture feedback mechanism.

Emotipix users have the option for extended interaction. To adapt to the dynamic mobile usage context, we should provide opportunities for users to interact more extensively with the system when desired, for example, when they have time and attention available. In addition, the user has the obvious possibilities for communication directly on the phone.

\subsection{User interface design}

When a user captures a new photo, Emotipix displays a photo upload form (Figure 1a). The form does not require any response; if the user does not interact with the form, the photo will be automatically published after a timeout period has elapsed. The user can optionally stop the timer, add a caption, or cancel a photo upload.

The primary interface for viewing photos is the phone's home screen, called the "Today Screen" on Windows Mobile (Figure 1b). Emotipix photos appear in the background of the home screen as the wallpaper, and are updated once per minute to create an automated 
slideshow of shared photos. A default interval of oneminute was chosen to provide regular updates without adversely affecting the phone's performance; users can also configure this parameter. Because the user interface is small, we display one full-screen photo at a time. The automated slideshow enables users to passively view photos, at the risk of missing photos; to mitigate this risk, photos are displayed repeatedly and can be browsed on the phone or the web.

A sticker (i.e., a tiny photo icon) superimposed on the lower left corner of each photo identifies the photo's publisher. Stickers superimposed along the lower right edge of a photo identify subscribers who have sent karma, (i.e., positive feedback), to the photo. Additionally, a caption, if provided, is superimposed. Thus, with a glance at the phone's home screen a viewer can see the photo, the publisher, the subscribers who have sent karma, and the caption. We superimpose this information to efficiently use the small display and to enable users to attune to it in the periphery. Stickers and captions are permanently affixed to photos. When a publisher sends karma to his/her own photo or when a user sends karma to a photo after having previously sent it karma, no stickers are affixed.

Users can interact with Emotipix via a small peace sign icon on the lower edge of the home screen. Tapping the icon associates karma with a photo: (1) permanently affixes the user's sticker in the corner of the photo (Figure 1c), (2) re-displays the photo (with sticker) to the user's buddies, and (3) increases the probability that the photo will be displayed in the future. Additionally, a tap-and-hold gesture on the Emotipix icon brings up a context menu. With this menu, users can adjust the rate of wallpaper updates, save a local copy of a photo, browse through an archive of cached photos, or initiate a phone call, text message, or picture message to a photo's publisher.

\subsection{Implementation}

Photo Publication. Emotipix employs simple channel-based publish/subscribe, managed by a server, for sharing photos. Each user has a single publication channel. Publishers are subscribed to their own channels, and subscriptions are symmetric. The Emotipix client automatically detects new photos by watching the phone's file system and uploads them to the server, where they are stored in a PostgreSQL database. The Emotipix server also provides a web interface, which enables users to upload any digital photos to Emotipix.

Client and Server. The Emotipix client, written in C\# using the .NET Compact Framework, uploads new photos and karmas to the server. It also periodically downloads photos from the server and maintains an in- memory 16-photo cache, using a policy of replacing the longest-residing photo. The client communicates with the server, also written in C\# using .NET, via asynchronous web services. During network lapses, the client displays cached images, and it retries failed communications after connectivity is restored.

Photo Prioritization. The Emotipix server maintains an outbound photo queue, for each subscriber, from which clients download photos. When a photo is published or receives karma, it is immediately enqueued for download. When a client requests a photo download for a subscriber whose queue is empty (i.e., no new photos or photos that just received karma are awaiting download) the server selects a photo from the database. A given photo's probability of selection decreases with age and increases with karma.

\section{User study}

We investigated the use of Emotipix through an exploratory 14-day user study with 16 participants, including 6 pairs and a 4-person group. Our study was designed to shed light on the following questions:

- Does Emotipix engage the periphery to facilitate social awareness and conversation?

- What practices emerge from use of Emotipix?

- What lessons does use of Emotipix provide for mobile peripheral display design?

\subsection{Participants}

To investigate social awareness and communication, we recruited pairs and small groups of close friends or family to participate in the study. We recruited 16 participants (12 living in San Diego and 4 in other US cities), via word of mouth, e-mail, and the web. They included 7 women and 9 men, ranging in age from 20 to 51 (median 25). They comprised six pairs (labeled A-F) and one group of four (labeled G); while the majority of our data pertains to pairs, we include data from the 4-person group to provide a counterpoint.

Group A was a married couple, a female student and a male engineer, in their twenties. Group B was a pair of co-workers, male and female, who were scientists in their early twenties. Pair C consisted of two male friends, a teacher and an engineer, in their forties. Group D was a couple in their twenties, a male graduate student living in San Diego and a female software engineer living in Redmond, Washington. Group E was a couple in their thirties, a male electric distribution analyst and a male caterer. Group F consisted of a mother and son, a 51 year old field services specialist and a 20 year old undergraduate student. Group G, four friends in their twenties, consisted of three women 


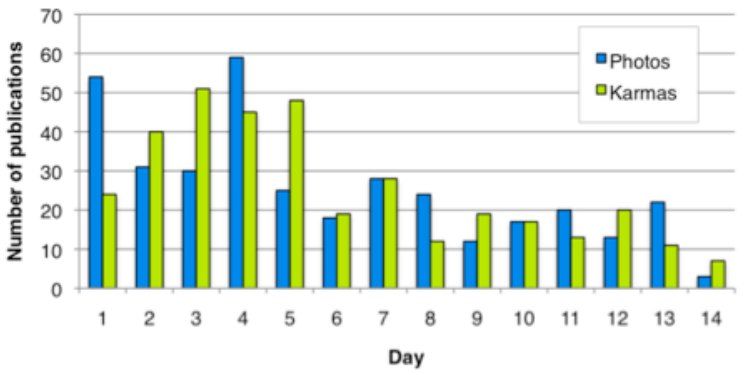

Figure 2. Publications by day

living in the San Francisco area and one man living in San Diego. The group included an environmental scientist, a programmer, a volunteer program assistant, and a business development intern. $\langle\mathrm{G} 1,<\mathrm{G} 3>$, and $<\mathrm{G} 4>$ were close friends. $<\mathrm{G} 2>$ was close friends with $<\mathrm{G} 4>$ and acquainted with $<\mathrm{G} 1>$ and $<\mathrm{G} 3>$.

\subsection{Hardware and software setup}

We provided each participant with an HP Mobile Messenger hw6945 Pocket PC phone running Emotipix. The phones have a $1.3 \mathrm{MP}$ camera, a $240 \times$ 240 pixel touch screen, a stylus, and a QWERTY keypad. They have $50 \mathrm{MB}$ of storage, $48 \mathrm{MB}$ of memory, and have GPRS and Wi-Fi connectivity.

Participants were asked to use these phones in place of their own mobile phones. To minimize disruption to participants, we transferred their SIM cards and contacts to the provided phones. Participants all signed up for, and were reimbursed for, unlimited GPRS data service during the study.

\subsection{Data collection}

For data collection we employed questionnaires, interviews, experience sampling, diaries, and logging. At the study start, we conducted brief training sessions, collected demographic information, and asked participants to select nicknames and sticker images to represent themselves. The Emotipix client and server logged usage. We also captured some usage context with the Experience Sampling Method (ESM) [5]; on randomly chosen uses of a phone, the client displayed a multiple-choice survey, which asked why the person was using the phone and what they were doing at the time. Participants were also asked to $\log$ in daily to a web diary to report on their recent experiences with Emotipix. The diary interface displayed recent photos to assist recollection and elicit responses [35]. We instructed participants to use Emotipix and fill in the diaries at their discretion; they were not required to take photos or write in their diaries. At the end of the study we interviewed participants regarding their experiences.

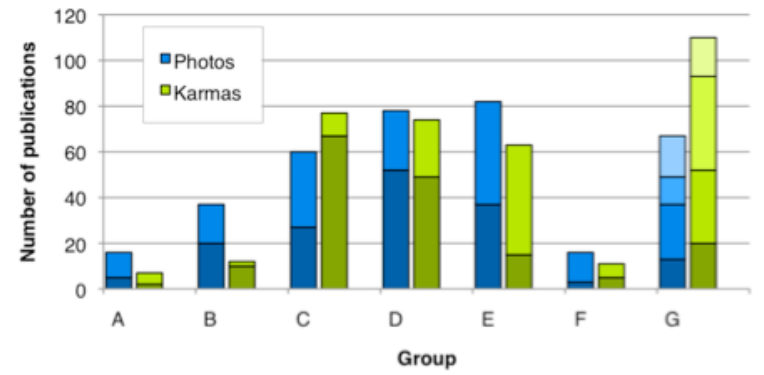

Figure 3. Publications by group

\section{Quantitative usage data}

During the two-week study, participants published 356 photos overall, 337 from the phones and 19 from the web. This amounts to an average of 22.3 photos per person $(\min 3, \max 52)$, or 1.6 photos per person per day. There was an average of 50.9 photos per group ( $\min 16, \max 82$ ), or 3.6 photos per group per day. There were 354 karmas given to 165 photos $(46.3 \%$ of published photos); images that received karma often received it more than once $(\max 12)$. Photographers sent 168 karmas to their own photos (in this case the photo's karma score increases but no sticker appears). Figure 2 shows daily publications, including photos and karmas, by group. Photo and karma activity was initially high, likely due to novelty effect, then leveled off, and finally dropped on the last day of the study, likely due to users' anticipation of the end of the study. Figure 3 shows total publications by group, broken down by participants (in ascending order by label).

The Emotipix integrated photo browser was used by many participants, and was used heavily by a few; we logged 6,820 navigational interactions (e.g., forward, backward). The other integrated options for extended interaction received limited usage (only Emotipix activity was logged); 8 photos were discussed via MMS, 10 via SMS, and 1 via a phone call, and 7 photos were saved on the phone.

There were 3232 ESM surveys displayed on the phone, with 1010 being answered and 2222 being cancelled or ignored. From the number of displayed surveys, triggered randomly on phone use, we see that people used their phones frequently and thus had many opportunities to see the peripheral display. From the survey responses, we determined that these uses were embedded in a variety of contexts. The top three reasons given for using the phone were checking calls and messages (36\%), calling or sending text messages $(20 \%)$, and checking the time $(13 \%)$, and the top three activity contexts were working or studying (31\%), transit (16\%), and killing time (14\%). 


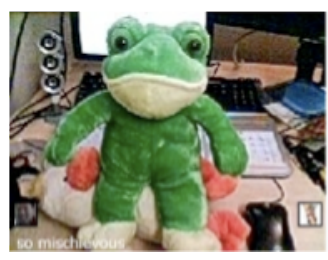

(a)

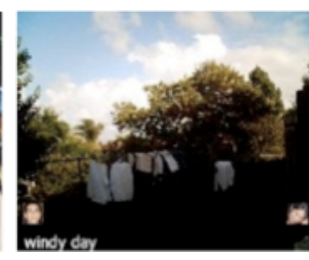

(b)

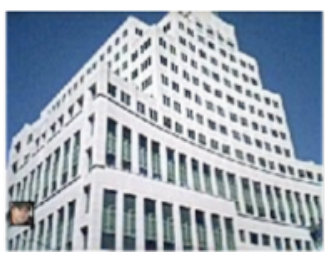

(c)

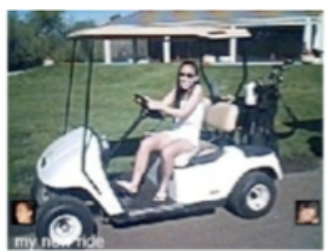

(d)

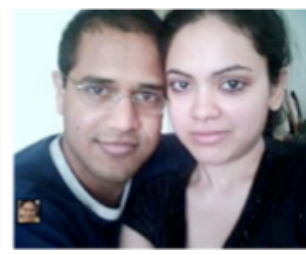

(e)

Figure 4. (a) Easing conversation (captioned "so mischievous"), (b) Sharing the mundane ("windy day"), (c) Aesthetics, (d) Sharing experiences ("my new ride"), (e) Gift giving

\section{Results}

We now turn to the qualitative data to gain insight into participants' interactions, as they pertain to communication and social practices. We analyzed the interview, diary, and photo data using elements from grounded theory [14], such as inductive coding. We first describe the participants overall experiences, i.e., how they interacted with and perceived Emotipix, and how Emotipix stimulated conversation. Then, we describe the topics of conversation and elements of conversational structure evinced in the use of Emotipix.

\subsection{Overall experiences}

Emotipix's display engaged the periphery. The Emotipix display engaged the periphery of users' attention. All 16 participants reported in interviews that, on the whole, they did not find Emotipix's display distracting or disruptive to their workflow, even while regularly noticing it. $<$ B2 $>$ offered a typical comment: "I took a second to glance at it, no big deal to me." Seemingly because the mobile phone has replaced the wrist watch for many people, participants commonly reported seeing pictures while checking the time: "I would look at the time and then I would be like, 'Oh, Emotipix is there" (D2). Participants also reported noticing images before and after sending or receiving calls and text messages, checking for missed calls or messages, and using other applications. They appreciated seeing the pictures frequently in the periphery, rather than having to explicitly seek them out: "When I picked up the phone to do something I'd see all the pictures... which I think is a nice thing, because to launch an application and having to view, you'd probably do it much less" (A2). Occlusion could occasionally cause distraction. $<\mathrm{C} 2\rangle$ said, "It's distracting when a picture shows up and I think, 'What does that word say? I can't read the caption."” To avoid this issue, the captioning mechanism could adapt to the photo's background color.

Despite the small size of the stickers persistently overlaid on photos, all participants reported that the stickers effectively conveyed information regarding who published or sent karma to photos. 14 participants reported that they noticed and enjoyed when their photos received karma. Karma was generally interpreted as an indication that one's friend had seen and liked a picture: "I liked it when a picture I took was appreciated, especially if I took a lot of effort to take the photo" (B1). Karma stickers could also convey affect: "I was happy when $<D 1>$ karma'd pics I took. I could imagine her smiling when she saw those pictures" (D2). In the 4-person group, in addition to identifying the sender, karma could act as a recommendation and prompt a transition from the periphery to the center of attention: "It made me pay more attention to pictures which had already been karma'd" (G3).

Emotipix reduced the effort for conversation.

All participants reported that Emotipix's automatic publication reduced the effort required to send photos, the peripheral display reduced the effort required to view photos, and the karma mechanism reduced the effort required to provide feedback. Overall, we found that Emotipix effectively eased conversation. Most participants reported using photos to visually communicate on their phones more than they did previously, as with Flipper, SLAM, and MMM2 [7, 8, 34], and expanding their definitions of which photos are worth sharing [28]. For example, $<$ D2 $>$ enjoyed exchanging photos, with his girlfriend, of stuffed animals in funny poses (Figure 4a). He explained, "I would never send her a picture message of that because it's just - it's not like, 'Oh my God, you have to see this.'... I feel like [MMS is] for really important things." $<\mathrm{C} 2>$ liked knowing that publishing photos would not disrupt his friend: "I don't have to bother him or interrupt him... I could just do this visually". $<$ B1 $>$ appreciated the ease of sending karma: "It's cool you can just tap one quick tap and you're like, 'Oh that's a cool picture. ,",

Because Emotipix required so little effort, there were a few occasions when people published photos or sent karma unintentionally. Accidental disclosure can have negative consequences. $\quad<\mathrm{B} 1>$, who shared pictures with a colleague, related: "It's a little scary that you cannot delete photos after the fact. I was worried the picture of my tattoo might show too much but it 


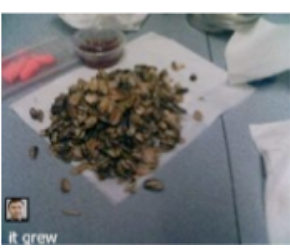

(a)

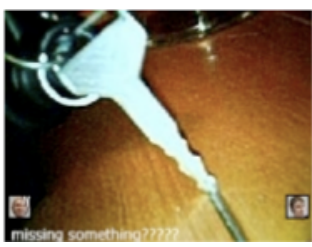

(b)

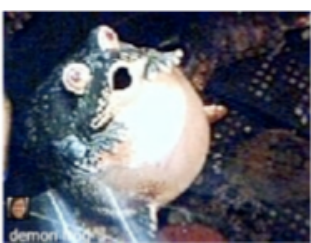

(c)

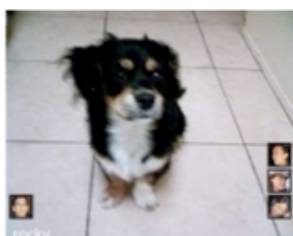

(d)

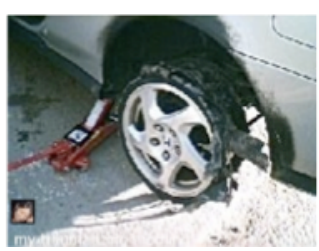

(e)

Figure 5. (a) Humor (captioned "it grew"), (b) Explicit communication ( "missing something?????"), (c) Second turn of an adjacency pair ("demon frog"), (d) Multiple karmas ("rocky"), (e) Expecting a response ("my traumatizing morning")

turned out ok." This anecdote highlights the need for error avoidance, detection, and repair mechanisms.

In addition to reducing the effort for conversation within the application, Emotipix stimulated conversation outside the application, with photos acting as topical resources: "When we get together after several days, we seem to laugh about the pictures we've been sharing" (C2). Emotipix also triggered conversations beyond our participant groups: "It became something to talk about. You know, showing my friends the wallpaper" (B2).

\subsection{Topics of conversation}

We developed a taxonomy, through inductive coding of the photo data, to categorize the subjects and uses of Emotipix photos. We were unable to directly use any existing taxonomies, such as Voida and Mynatt's themes for communicative appropriation of photos in instant messaging [36]. Their themes do not cover all practices we observed (e.g., "sharing experiences"), and some are not applicable to Emotipix (e.g., "image as amplification" of accompanying text). We describe participants' practices with each category (N.B., each photo could be labeled with more than one category).

Sharing the mundane. $49 \%$ of photos depicted aspects of everyday life, such as homes, workplaces, pets, family members, weather (Figure $4 \mathrm{~b}$ ), and meals. $<\mathrm{A} 2>$ explained, "I'm not taking these pictures for me to look at later and say, 'Hey, I was working.' It is just to know that somebody else is looking at it and they know how I'm doing." These mundane photos often conveyed an implicit status update or "thinking of you" message, and merely the act of sharing a photo was meaningful. Kuwabara et al. note that sharing implicit information, such as presence and status, may foster social connectedness [20]. Viewing mundane photos also helped participants feel more aware of friends' activities: "I like how it definitely gave me a glimpse more into their everyday life and just what was going on around them if we couldn't physically be together" (G3).
Aesthetics. $11 \%$ of the photos were taken specifically for their aesthetic value, or beauty. Because Emotipix presents photos in a mobile phone display's background, as one might expect, some photos were taken to serve decorative purposes: "I thought this would make a good wallpaper" (B1). Other photos captured moments of natural beauty (e.g., a sunset) and artistic compositions (Figure 4c).

Sharing experiences. $25 \%$ of Emotipix photos depicted an experience that the photographer intended to share with his or her partner(s). Although essentially all photos were taken with the intent of sharing, due to Emotipix's automated publication we limit this category to pictures of specific experiences. We included practical activities (e.g., $<\mathrm{E} 2>$ going house hunting), serendipitous encounters (e.g., $<$ D $2>$ seeing a huge inflatable birthday cake on campus), and other non-mundane events (e.g., $<\mathrm{G} 4>$ playing golf for the first time, shown in Figure 4d). Interestingly, 3\% of all photos illustrated and invited future shared experiences. For instance, $<\mathrm{D} 1>$ sent $<\mathrm{D} 2>$ a picture of a video game she had just purchased, suggesting that they play it together upon his next visit.

Gift giving. 7\% of photos evinced gift giving, i.e., social exchange, practices among Emotipix users. Although many Emotipix photos could be considered gifts, as defined in Taylor and Harper's study of SMS use among teenagers [33], we limit this category to photos that were explicitly intended as such. For example, the couples in our study sometimes published pictures of themselves, alone or together (Figure 4e), as gifts. $<\mathrm{A} 1>$ sent several self-portraits to her husband $<\mathrm{A} 2>$, who said: "...I feel good when I get a picture [...] of $\langle A 1\rangle$ just at home, just the part that she thought of taking a picture and sending me is like a nice thing". Another type of gift giving that occurred mainly among the non-couples involved disclosing personal information, for example, sharing pictures of pets, tattoos, or one's parents' house.

Humor. $27 \%$ of Emotipix photos contained humor, provoking laughter or amusement. $15 \%$ of the humorous pictures contained specific teasing, and $60 \%$ of the humorous photos were inside jokes. For example, 
$<$ B2 $>$ took photos of a growing pile of sunflower seed shells throughout one day to show $<\mathrm{B} 1>$ how many seeds he was eating, because she had recommended that particular snack to him (Figure 5a). Almost half (47\%) of the humorous photos were elements of running jokes. When $<\mathrm{G} 1>$ was stuck in traffic, she took a picture of her car's speedometer at zero, captioned "hooow fast I am going" and her friend $<\mathrm{G} 4>$ posted a similar photo several days later.

Explicit communication. We did not expect Emotipix to be used for explicit communication conveying specific information, since publishers could not guarantee if or when subscriber(s) would see photos. Yet, $5 \%$ of the photos fell into this category. $<$ E2 $>$ published several photos of houses, which he looked at with a real estate agent, for his partner to see. Also, after $<\mathrm{C} 1>$ accidentally left his keys at $<\mathrm{C} 2>$ 's house, $<\mathrm{C} 2>$ published a photo of the keys (Figure $5 \mathrm{~b}$ ), captioned "Missing something?".

\subsection{Conversational structure}

Participants' communicative practices with Emotipix evinced elements of conversational structure, including turn-taking and balanced contribution [22]. Participants also attempted to steer conversations toward topics of mutual interest.

Participants took turns publishing photos. For instance, $<$ B2 $>$ published a photo in which his girlfriend's dog's eyes appeared to glow in the dark, captioned "demon dog". $<$ B1 $>$ responded with a picture of a funny-looking ceramic frog statue, captioned "demon frog" (Figure 5c). Within a conversational thread, the photos' topical coherence was sometimes sufficient to indicate their relationship, and as in this example, captions could be used to provide more explicit references. A single conversational turn could consist of a sequence of photos. On one occasion, $<\mathrm{C} 2>$ published 9 photos in quick succession, constructing a narrative intended to convince $<\mathrm{C} 1>$ to go surfing.

Although the order of messages is an important element of conversational structure, order is not preserved in the Emotipix client. New photos are displayed in chronological order, but older photos are not. The original order is, however, preserved in the web interface to the photo archive. None of the participants commented on the lack of persistent ordering or requested system support for it.

A conversational turn could also consist of sending karma. A publisher may respond to karma by incorporating the feedback in deciding what photos to publish. $<\mathrm{G} 2>$ 's picture of his dog (Figure $5 \mathrm{~d}$ ) received karma from the other three members of his group, and he responded by publishing several similar pictures. Because a subscriber's karma sticker can appear only once on a photo, and publishers cannot directly respond, Emotipix avoids the incessant back-and-forth clicking described in Kaye's study [17]. A subscriber may also respond to another subscriber's karma. For instance, $<\mathrm{G} 2>$ published a photo of his new pair of shoes to show them off, and $<\mathrm{G} 3>$ sent karma to his photo to express her approval. $<\mathrm{G} 4>$ hoped to avoid further conversation on that topic so she withheld a response and encouraged $<\mathrm{G} 1>$ to do the same.

Turn taking, along with a sense of reciprocal involvement, motivated continued use of Emotipix by confirming that the conversation was balanced. Participants often felt compelled to reply to their partners' photos, to complete adjacency pairs. For example, $<\mathrm{D} 1>$ sent photos to provoke her partner to respond in kind: "Sometimes it's just 'cause like I'm wondering what $<D 2>$ 's doing, so I just send him a random picture and then if he sends me a random picture, then I can kinda tell" (D1). A satisfactory response could take several forms: "I think it is a good incentive to take a lot of pictures if there is enough feedback... and that feedback could be a direct comment about the picture itself or them taking more pictures" (G2). Beyond the scale of individual messages, participants' practices reflected a desire for reciprocity, and social pressure was sometimes applied to encourage it. For example, early in the study $<\mathrm{G} 1>$ published a picture of $\langle\mathrm{G} 4>$ captioned, "lame-o with no pics" because she had not published any Emotipix photos yet.

\section{Mobile peripheral display design}

Our study of Emotipix, an application that exploits the periphery on mobile phones, provided not only valuable insights into potential uses of such systems, but also potential complications of their use. Common to the following complications is a theme of tension between peripherality and control.

\subsection{System behavior vs. users' expectations}

Some participants falsely expected Emotipix to behave like the existing, familiar communication mechanisms (e.g., SMS, MMS, email) that mobile phones typically support. In fact, they often described Emotipix photo publication as analogous to sending email or text messages: "The pictures kind of substituted for text messaging for me" (D1).

Complications could arise when Emotipix did not work as participants expected. For example, although we informed them that Emotipix did not guarantee if or when subscribers would see specific pictures, photographers expected their friends to see new pictures promptly. $<\mathrm{G} 4>$ published a picture of her 
blown-out car tire, and she became upset that $<\mathrm{G} 2>$ did not call her afterwards to ask how she was doing (Figure 5e). $<\mathrm{G} 2>$ recalled, "I actually got in trouble for not asking, because I didn't see the picture... And I got [a text message] that said 'Oh, thanks for not asking if I almost died.' I'm like 'Why? I didn't know. "'

To mitigate these issues, as designers we should attempt to better manage users' expectations. For example, because Emotipix introduces ambiguity into visual communication (i.e., one cannot know if someone has seen a photo unless they provide explicit feedback), the system could indicate this ambiguity explicitly (e.g., by indicating which buddies may not have seen a particular photo).

\subsection{Users want control}

Sometimes participants used social mechanisms to augment and control the system's behavior. Two participants used Emotipix's photo browser to ensure that they saw all new photos. $\langle\mathrm{C} 2>$ said, "I would constantly go to Emotipix... and then cycle through all 16 [cached] pictures to see what I had missed." Photographers sometimes alerted friends of new photos: "You knew that eventually they would see it... You still wanted to get that message out - to text or call, 'Hey check out my new pics"' (E1). Also, many participants wanted more control over which photos were displayed, so they repeatedly sent karma to their favorites. $<\mathrm{C} 1>$ explained, "I karma'd the surfing ones 'cause - I really karma'd them because I wanted to see 'em again."

In many respects these practices, which complement Emotipix's intended calmness, are in line with Rogers' assessment of how users appropriate technologies for their own ends [30]. Yet we do face a design challenge: how can we give users the control they desire without increasing unwanted distraction or requiring undue effort? Adding features and buttons could detract from the interface's simplicity and ease of use, yet as Weiser pointed out, providing users with a sense of control is key to providing them with a sense of calm. We believe that a small number of additional buttons on the home screen and the upload screen, as well as a few offline configuration parameters, can help strike a balance among the tradeoffs involved.

\subsection{Privacy}

Any communication system has the potential for privacy problems because the recipient may share information in ways not desired by the sender. Emotipix expands the potential for undesired sharing in two ways. First, photos are automatically broadcast to all buddies, so the sender may not fully calculate the possible consequences of releasing a particular photo.
Users may learn to mitigate this problem by limiting their inner circles, as is happening now with social networking applications like Facebook. Second, photos are displayed automatically, rather than at the receiver's choosing; and although the mobile phone is a personal device, it is possible that non-buddies will see photos on the phone's display when it is in use. However, the small size and narrow transmission angle of a phone's display minimize the potential for accidental viewings.

In line with enhancing user control, Emotipix could provide customized sub-channels for publishing (e.g., "family", "friends"). On the recipient side, display controls or filters could be provided akin to the various ring modes that phones now provide. Such features could enable the system to scale beyond small, cohesive, high-trust groups.

\section{Conclusion}

Our exploratory study of the use of Emotipix provides insights into the types of mobile communication for which the periphery is well suited. We have learned that a communication mechanism that engages the periphery on mobile phones is effective for supporting social awareness, connectedness, and conversation in a non-disruptive, calm manner. It provides opportunities for lightweight and more extended interaction without requiring their use. Additionally, a mobile peripheral visual display can introduce interesting ambiguity and serendipity into the experience; one does not know if, when, or where information will be seen. A communication mechanism that employs such an interface can lower perceived barriers and stimulate conversation; yet at the same time, this mechanism is not appropriate for conveying time-dependent, critical, or private information.

We have also learned lessons applicable to the design of interfaces that engage the periphery in mobile visual communication. First, when introducing a new communication mechanism, we need to manage users' expectations as they learn to incorporate it into their existing practices. Second, we must manage the tension between enabling users to control the system's behavior and not obligating them to do so. Third, when we reduce the effort required to send and receive messages we should enable users to manage their privacy.

\section{References}

[1] Barkhuus, L., Brown, B., Bell, M., Sherwood, S., Hall, M. and Chalmers, M. From awareness to repartee: Sharing location within social groups. Proc. CHI 2008, ACM Press (2008), 497-506.

[2] Brown, B., Taylor, A., Izadi, S., Sellen, A., and Kaye, J. Locating family values: A field trial of the Whereabouts Clock. Proc. Ubicomp 2007, Springer-Verlag, 354-371, 2007. 
[3] Chang, A., Resner, B., Koerner, B., Wang, X. and Ishii, H. LumiTouch: An emotional communication device. Ext. Abstracts CHI 2001, 313-314, 2001.

[4] Consolvo, S., McDonald, D. W., Toscos, T., Chen, M. Y., Froehlich, J., Harrison, B., Klasnja, P., LaMarca, A., LeGrand, L., Libby, R., Smith, I., and Landay, J. A. Activity sensing in the wild: a field trial of ubifit garden. Proc. CHI 2008. ACM Press (2008), 1797-1806.

[5] Consolvo, S. and Walker, M. Using the Experience Sampling Method to Evaluate Ubicomp Applications. Pervasive Computing 2, 2 (2003), IEEE, 24-31.

[6] Consolvo, S., Everitt, K., Smith, I., and Landay, J. A. Design requirements for technologies that encourage physical activity. Proc. CHI 2006, ACM Press (2006), 457-466.

[7] Counts, S., Fellheimer, E. Supporting social presence through lightweight photo sharing on and off the desktop. Proc. CHI 2004, ACM Press (2004), 599-606.

[8] Counts, S., Group-Based Mobile Messaging in Support of the Social Side of Leisure. Proc. CSCW 2007, 75-97.

[9] Dey, A. K. and de Guzman, E. From awareness to connectedness: the design and deployment of presence displays. Proc. CHI 2006. ACM Press (2006), 899-908.

[10] Dourish, P. and Bly, S. Portholes: supporting awareness in a distributed work group. Proc. CHI 1992, ACM Press (1992) 541-547.

[11] Froehlich, J., Dillahunt, T., Klasnja, P., Mankoff, J., Consolvo, S., Harrison, B., Landay, J. UbiGreen: Investigating a Mobile Tool for Tracking and Supporting Green Transportation Habits. Proc. CHI 2009, ACM Press (2009).

[12] Frohlich, D., Kuchinsky, A., Pering, C., Don, A., and Ariss, S. Requirements for Photoware. Proc. CSCW 2002, ACM Press (2002), 166-175.

[13] Garau, M., Poisson, J., Lederer, S., and Beckmann, C. Speaking in Pictures: Visual Conversation Using Radar. PICS Workshop, Ubicomp 2006.

[14] Glaser, B., Strauss, A. The discovery of grounded theory: Strategies for qualitative research. 1977.

[15] Greenberg, S. and Rounding, M. The notification collage: posting information to public and personal displays. Proc. CHI 2001, ACM Press (2001), 514-521.

[16] Ishii, H., Ren, S. and Frei, P. Pinwheels: visualizing information flow in an architectural space. Ext. Abstracts CHI 2001, ACM Press (2001), 111-112.

[17] Kaye, J., I just clicked to say I love you: rich evaluations of minimal communication, Ext. Abstracts CHI 2006, ACM Press (2006).

[18] Kindberg, T., Spasojevic, M., Fleck, R., and Sellen, A. The ubiquitous camera: An in-depth study of camera phone use, Pervasive Computing 4, 2 (2005), IEEE, 42- 50.

[19] Kirk, D., Sellen, A., Rother, C., and Wood, K. Understanding photowork. Proc. CHI 2006, ACM Press (2006), 761-770

[20] Kuwabara, K.; Watanabe, T.; Ohguro, T.; Itoh, Y.; Maeda, Y., Connectedness oriented communication: Fostering a sense of connectedness to augment social relationships. Proc. SAINT 2002, IEEE, 186-193.

[21] Liechti, O. and T. Ichikawa. A digital photography framework enabling affective awareness in home communication. Personal Technologies 4, 1 (2000).

[22] Littlejohn, S.W. and Foss, K.A. Theories of Human Communication. Thomson Wadsworth, USA. 2004.

[23] Mankoff, J., Dey, A.K., Hsieh, G., Kientz, J., Lederer, S. and Ames, M. Heuristic evaluation of ambient displays. Proc. CHI 2003, ACM Press (2003), 169-176.

[24] Markopoulos, P., Romero, N., Van Baren, J., IJsselsteijn, W., Ruyter, B., and Farshcian, B. Keeping in touch with the family: Home and away with the ASTRA awareness system. Ext. Abstracts CHI 2004, ACM Press (2004), 1351-1354.

[25] Mynatt, E.D., Back, M., and Want, R. Designing audio aura. Proc. CHI 1998, ACM Press (1998), 566-573.

[26] Mynatt, E. D., Rowan, J., Craighill, S., and Jacobs, A. Digital family portraits: Supporting peace of mind for extended family members. Proc. CHI 2001, ACM Press (2001), 333-340.

[27] Naaman, M., Nair, R., Kaplun, V. Photos on the go: A mobile application case study. Proc. CHI 2008, ACM Press (2008).

[28] Okabe, D. and Ito, M. Camera phones changing the definition of picture-worthy. Japan Media Review, 2003.

[29] Okabe, D. Emergent social practices, situations and relations through everyday camera phone use. Proc. Int'l Conf. on Mobile Comm. and Social Change, 2004, 1-19.

[30] Rogers, Y. Moving on from Weiser's vision of calm computing: Engaging UbiComp experiences. Proc. UbiComp 2006, IEEE Press (2006), 404-421.

[31] Sit, R. Y., Hollan, J. D., and Griswold, W. G. Digital Photos as Conversational Anchors, Proc. HICSS 2005, 109119.

[32] Smith, I., Consolvo, S., Hightower, J., Hughes, J., Iachello, G., LaMarca, A., Scott, J., Sohn, T., Abowd, G., Social disclosure of place: From location technology to communication practice, Proc. Pervasive 2005, Springer (2005), 134-151.

[33] Taylor, A. S. and Harper, R. Age-old practices in the 'new world': A study of gift-giving between teenage mobile phone users. Proc. CHI 2002, ACM Press (2002), 439-446.

[34] Van House, N., Davis, M., Ames, M., Finn, M., and Viswanathan, V. The uses of personal networked digital imaging: An empirical study of cameraphone photos and sharing. Proc. CHI 2005, ACM Press (2005), 1853-1856.

[35] Van House, N. A. Interview viz: Visualization-assisted photo elicitation. Ext. Abstracts CHI 2006, ACM Press (2006), 1463-1468.

[36] Voida, A. and Mynatt, E. D. Six themes of the communicative appropriation of photographic images. Proc. CHI 2005, ACM Press (2005), 171-180.

[37] Weiser, M. and Brown, J. S. Designing calm technology. PowerGrid Journal 1, 1, July 1996. 